\title{
Numerical issues in plasticity models for granular flows
}

\author{
Pierre A. Gremaud ${ }^{1}$ \\ Department of Mathematics and Center for Research in Scientific Computation, \\ North Carolina State University, Raleigh, NC 27695-8205, USA
}

\begin{abstract}
Friction plays a fundamental role in the Mechanics of granular materials. Two problems are considered: (i) heap formation, (ii) granular flow. Both problems admit closely related mathematical models. In each case, analytical and numerical difficulties are discussed. Efficient and reliable numerical methods are proposed and implemented. The results are illustrated by several computational experiments.
\end{abstract}

Key words: granular material; plasticity; heap formation; granular flow; numerical methods

\section{Introduction}

The handling of granular material poses several important and challenging problems. In industrial applications for instance, silos routinely malfunction; other unwanted effects may include segregation in powders or disintegration of brittle particles while being flown down a chute, to mention but a few. The chemical, mining and pharmaceutical industries, among others, incur very significant financial losses as

Email address: gremaud@unity.ncsu.edu (Pierre A. Gremaud).

1 Partially supported by the Research Office (ARO) through grant DAAD1999-1-0188 and by the National Science Foundation (NSF) through grants DMS-9818900 and DMS-02044578.

Preprint submitted to Elsevier Science a result of such phenomena. Granular material mechanics also plays a fundamental role in countless geophysical phenomena.

While most, but not all, applications are time dependent, the time dependent versions of most plasticity models for granular flows are hopelessly illposed. Typically, equations analogous to $\partial_{t} u=\partial_{x x} u-\partial_{y y} u$ are obtained (Schaeffer, 1987).

In this paper, several numerical difficulties linked to simple models for granular piles (Section 2) and granular flows (Section 3) are discussed. The models, which are fairly classical, are described in a two-dimensional setting, in order to focus the discussion on the difficulties at hand. The presented numerical results are however fully three-dimensional. 


\section{$2 \quad$ Steady state piles}

When a dry granular material is poured on a surface, the slope of the resulting pile cannot exceed some critical value: the angle of repose. The pile takes a conical shape, such as for instance that of sand in the lower part of an hour glass. Related phenomena also contribute to the shape and formation of cinder cones, although this type of application is not considered here per se. We focus on how to determine the shape of such piles when obstacles are present, see Figure 2. A related and deceptively simple industrial problem is the determination of the volume of material inside a bin of possibly complicated geometry including inserts, internal walls, etc... In practice, this is done by having one or several gauges measuring the height of the material at given points. Without some knowledge of the geometry of the top free boundary, such methods are inaccurate.

The problem of finding the shape of a granular heap has a long history, largely due to its connection to soil mechanics in civil and military engineering. Coulomb (1776) was the first to relate the angle of slip to the friction properties of the material. Unlike previous work, he did not assume a priori values of that angle, see Heyman (1972) for historical background.

The equilibrium equations are

$\nabla \cdot T=\rho g$

where $T$ is the stress tensor, $\rho$ is the density, assumed to be constant and $g$ is the acceleration due to gravity. The constitutive laws are essentially a multidimensional counterpart to the standard Coulomb's law of dry friction. Unfortunately, there is more than one meaningful way of generalizing this law from one to several space dimensions. A standard model corresponds to the von Mises yield condition (Nedderman, 1992). In a two-dimensional setting (to simplify this introduction), it reads

$\left|\frac{\sigma_{1}-\sigma_{2}}{\sigma_{1}+\sigma_{2}}\right| \leq \sin \delta$

where $\sigma_{i}, i=1,2$, are the eigenvalues of the stress tensor $T$ (principal stresses) and $\delta$ is the angle of internal friction ${ }^{2}$.

Coulomb's argument is not complete in that, among other things, it does not describe the onset of avalanches. Further, it is assumed that no material gets into free fall, off an obstacle for instance. This places some geometrical restrictions on the problem. Whereas Coulomb's theory predicts a constant slope of the heap surface, experiments reveal that the slope may vary slightly. For a growing heap, the slope may become time dependent (Grasselli and Herrmann, 1999). Impact of falling particles at the top and interaction with walls at the bottom may also result in departure from constant slope (Grasselli and Herrmann, 2001). Various models have been proposed to account for those variations (Bagnold, 1941, Behringher, 1993, Bouchaud et al., 1995, Boutreux et al., 1997). Here, the angle of repose is assumed to be constant and equal to the angle of internal friction, which is a reasonable approximation provided the material is not cohesive (Nedderman, 1992).

Consider a single source above the ori-

2 The angle of internal friction is a convenient way of describing the coefficient of internal friction $\mu$ of the granular material, since by definition, $\mu=\tan \delta$. 


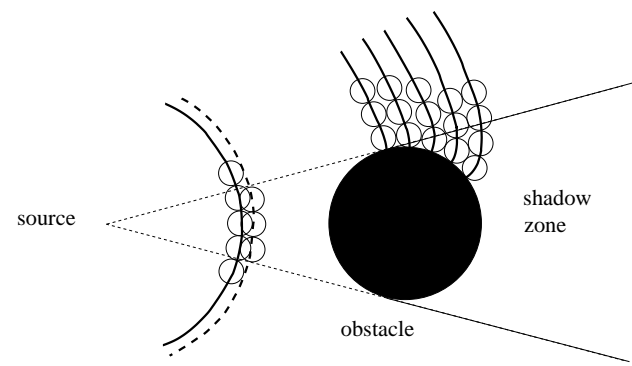

Fig. 1. The Huygens principle at play in the two dimensional case.

gin of the Cartesian coordinate system $(x, y, z)$ (several sources can handled by repeating the approach outlined below and postprocessing appropriately). The highest point of the pile is directly below the source and is denoted $(0,0, H)$. In the industrial problem, $H$ is measured and known at all time, and can be used as a parameter. In what follows, $\Omega \subset\{z>0\}$ represents obstacles and/or walls while $\Lambda=\{z>0\} \backslash \bar{\Omega}$ is the space that can be potentially occupied by the material. The problem is then, given $H>0$ such that $(0,0, H) \in$ $\Lambda$, find the part of $\Lambda$ that is occupied by the material.

The problem can be recast as one of traveltime determination. Assume $\Omega$ is cylindrical, i.e., $\Omega=\omega \times \mathrm{R}^{+}$, where $\omega \subset$ $\mathrm{R}^{2}$. The main idea is to relate the height $u(x, y)$ of the material at a point $P(x, y)$ to the time $\tau(x, y)$ it would take a signal emited at the source $O$ to reach that point. The fixed slope $\tan \delta$ of the heap corresponds to a fixed rate of increase of $\tau$. Following this analogy, the speed of propagation is then $1 / \tan \delta$. The solution can be constructed according to the classical Huygens principle. The wavefront of a propagating wave emanating from $O$ conforms, at any instant, to the envelope of spherical wavelets emanating from every point on the wavefront at the prior instant. Figure 1, illustrates this principle in the case of a circular right cylindrical obstacle. In the visible part of the domain, i.e., at points that can "see" the source, the exact solution is a cone of half opening angle $\pi / 2-\delta$, hence the level lines are circular. In the shadow zone, those lines are bent as the wavefront turns around the obstacle.

To find the height of material at $P \in \mathrm{R}^{2} \backslash \omega$, the problem essentially boils down to finding the shortest distance $d(P, O)$ bewteen $P$ and the source $O(0,0)$, i.e.

$$
\begin{aligned}
d(P, O) & =\inf \left\{\int_{0}^{1}\left|\frac{d \gamma}{d s}(s)\right| d s, \gamma \in \mathrm{C}^{1}\left([0,1] ; \mathrm{R}^{2}\right),\right. \\
\gamma(s) \in R^{2} \backslash \omega & \forall s \in[0,1], \gamma(0)=O, \gamma(1)=P\} .
\end{aligned}
$$

This link between the height of the material and $d(\cdot, \cdot)$ while intuitively clear, can be formally established (Feldman, 1999). For some simple cylindrical obstacles, explicit solutions can be found by hand. Figure 2, illustrates the exact shape of the free surface around a circular cylindrical obstacle.

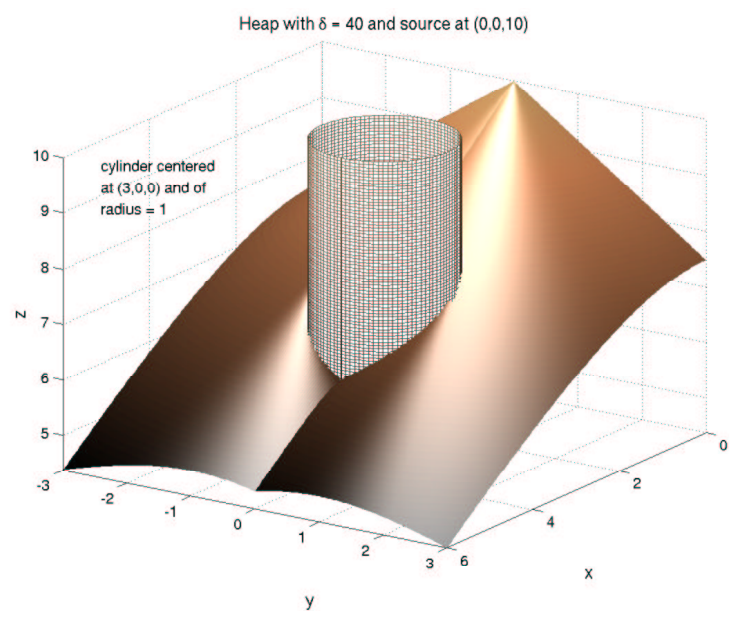

Fig. 2. Exact heap around a circular cylindrical obstacle for a material with an angle of repose of $\delta=40^{\circ}$

For general cylindrical obstacles, the efficient calculation of $d(\cdot, \cdot)$ is at the heart of the problem and is, by itself, still an area of research in com- 
putational geometry, see for instance Kapoor et al., 1997 and the references therein. Rather than attacking this problem directly, we propose an Eikonal formulation. The obstacle is characterized by the fact that the material (or wavefront) cannot penetrate it. Equivalently, this corresponds to a vanishing speed of propagation there. If $F$ denotes the slowness, i.e., the inverse of the speed, we set

$$
F(x, y)= \begin{cases}\tan \delta & \text { if }(x, y) \notin \omega \\ \infty & \text { if }(x, y) \in \omega\end{cases}
$$

The traveltime $\tau$ then satisfies

$$
\begin{aligned}
|\nabla \tau| & =F \quad \text { in } \mathrm{R}^{2}, \\
\tau(0,0) & =0 .
\end{aligned}
$$

We have therefore recast our problem into finding $\tau$ that solves $(2.3,2.4)$ from which the height $u$ is easily obtained through $u(x, y)=\max \{H-\tau(x, y), 0\}$. Equation (2.3) is the Eikonal equation; such a formulation is typical of traveltime problems, for instance related to geophysics (van Trier and Symes, 1991).

The Huygens principle can be viewed as a selection mechanism that picks among all the (weak) solutions to (2.3 , 2.4) the one that respects causality or, in other words, the fact that information propagates "one way". There is no mechanism in $(2.3,2.4)$ that ensures $\tau$ to go from smaller values to larger values when moving outwards from $O$ ("sawtooth" solutions are possible). The relevant solution is well known to be the viscosity solution (Crandall et al., 1983, 1984, Sethian, 1999).

Consider now a general three dimensional obstacle. In standard three dimensional traveltime problems (Sethian and Popovici, 1999), level sets of the time of propagation $\tau$ are surfaces and all spatial dimensions essentially play an identical role. Our problem is clearly different in that level sets for $\tau$ or the height $u$ are still curves, as in the previous two-dimensional case. The only difference between our two and three dimensional formulations is the role played by the obstacle. A parallel can still be drawn with the classical three dimensional problem if instead of considering a spherical propagation of the signal emited by the source, one considers a "cylindrical" mode of propagation. In other words, there is infinite speed of propagation in the $z$ direction ( $z$ cannot be interpreted as a pseudotime anymore). As before, one solves $(2.3,2.4)$ but now the slowness $F$ is itself a function of the unknown $\tau$, i.e.

$F(x, y, \tau)= \begin{cases}\tan \delta & \text { if }(x, y, u(x, y)) \notin \Omega \\ \infty & \text { otherwise }\end{cases}$

where $u(x, y)=\max \{H-\tau(x, y), 0\}$.

The design of efficient numerical methods for the resolution of HamiltonJacobi equations is a very active field of research, see for instance Crandall and Lions, 1984, Osher and Shu, 1991, Rouy and Tourin, 1992, Jiang and Peng, 2000, for "standard" approaches. Recently, a lot of attention has been focused on the design of fast methods. The Fast Marching Method (FMM) (Tsitsiklis, 1995, Sethian, 1999, Sethian and Popovici, 1999) is one example of such fast methods. It combines a heap sort algorithm with the so-called Dijkstra algorithm (Dijkstra, 1959) and has complexity $\mathcal{O}\left(N_{t o t} \log N_{t o t}\right)$ where $N_{t o t}$ is the total number of nodes. The Fast Sweeping Method (FSM) (Tsai et al., 2002, Zhao, 2002) is another promising example of a fast method. It combines a Godunov flux with a Gauss-Seidel 
like iterative process and has complexity $\mathcal{O}\left(N_{t o t}\right)$. In both cases however, several issues related to accuracy are unresolved.

As observed in Qian and Symes, 2002, in the present single source point case, a singularity of the traveltime field (and hence the height) is clearly located at the source itself. Therefore unless special care is taken near the source, a severe loss of accuracy results. Table 2 illustrates this phenomenon. Using a purely upwind second order FMM for solving a two-dimensional Eikonal equation with constant slowness leads to a method that is only roughly first order. Second order convergence is observed only if the exact solution is prescribed around the source in the mesh independent domain.

In general, prescribing the exact solution in a fixed neighborhood of the source may be problematic. For instance in case of a non constant slowness, the exact viscosity solution may not be available. In the present case the slowness is constant away from obstacles and thus, this heavy handed initialization step can be easily performed. However, when obstacles are present, points that naturally appear in the numerical stencil cannot be used. As a result, a loss in accuracy is to be expected. We refer to Ahmed et al., 2002 , for comments on how to combine "marchability" and accuracy by local adaption of the stencil. The issue of accuracy of the marching methods is in fact delicate due to the possible existence of accuracy barriers. For (linear) hyperbolic conservation laws, there is an extensive literature on optimal accuracy. For instance, it is well known that stable one-sided discretizations of $\partial_{y} u=\partial_{x} u$ can only be at most of order two (Iserles and Strang, 1983). Similar

\begin{tabular}{|l|l|l|l|l|}
\hline & \multicolumn{4}{|c|}{ 2nd order } \\
\hline $\mathrm{N}$ & $L^{2}$ & rate & $L^{\infty}$ & rate \\
\hline 50 & $6.48(-2)$ & & $1.83(-2)$ & \\
100 & $3.50(-2)$ & .89 & $9.38(-3)$ & .97 \\
200 & $1.81(-2)$ & .95 & $4.74(-3)$ & .99 \\
400 & $9.26(-3)$ & .97 & $2.38(-3)$ & .99 \\
800 & $4.67(-3)$ & .97 & $1.19(-3)$ & 1.0 \\
1600 & $2.35(-3)$ & .99 & $5.97(-4)$ & .99 \\
\hline & \multicolumn{5}{|c|}{ corrected $2 n d$ order } \\
\hline $\mathrm{N}$ & $L^{2}$ & rate & $L^{\infty}$ & rate \\
\hline 50 & $2.05(-2)$ & \multicolumn{4}{|c|}{$6.77(-3)$} & \\
100 & $4.96(-3)$ & 2.0 & $1.65(-3)$ & 2.0 \\
200 & $1.20(-3)$ & 2.0 & $4.07(-4)$ & 2.0 \\
400 & $2.96(-4)$ & 2.0 & $1.01(-4)$ & 2.0 \\
800 & $7.33(-5)$ & 2.0 & $2.51(-5)$ & 2.0 \\
1600 & $1.82(-5)$ & 2.0 & $6.26(-6)$ & 2.0 \\
\hline
\end{tabular}

Table 1

Comparison between the orders of convergence of FMM with corrected values, i.e., seeding of the exact in a neighborhood of the source and without.

result should also be expected to hold here, as FMM is a kind of one-sided method.

Figure 3 illustrates the efficiency of the method in the efficiency of the numerical method on a fully three dimensional case.

\section{Plastic flows}

In contrast to the static problems from the previous section, we now turn to simple granular flows. Following Schaeffer (1987) and the remarks in the Introduction only established, i.e., steady state, flows are considered. The govern- 


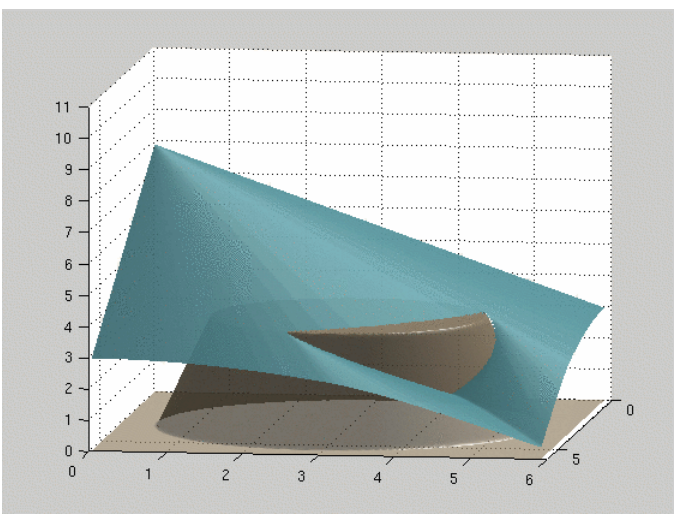

Fig. 3. Computed heap around a truncated conical obstacle for a material with an angle of repose of $\delta=40^{\circ}$

ing principles are conservation of momentum and mass. Those are respectively given by the equilibrium equation $\nabla \cdot T=\rho g,(2.1)$, from the previous Section, together with the equation of continuity

$\nabla \cdot v=0$

where $v$ is the velocity. In a twodimensional setting for instance ${ }^{3},(2.1$, 3.5 ) is a set of three scalar equations for five scalar unknowns, three components of the $2 \times 2$ symmetric stress tensor $T$ and two components of the velocity $v$. The system is closed by imposing two constitutive laws, a flow rule which links the stress tensor $T$ to the strain rate tensor and a yield condition, see Jackson, 1983, Schaeffer, 1987, Nedderman, 1992 for a full discussion. The yield condition corresponds to the fundamental assumption that the material has reached yield everywhere, i.e., it deforms everywhere. Mathematically, the inequality (2.2) is now an equality; it can be rewritten

\footnotetext{
3 Our study pertains to the full threedimensional case, a two-dimensional setting is only adopted for the purpose of exposition.
}

$\frac{\sigma_{1}(T)}{\sigma_{2}(T)}=\frac{1+\sin \delta}{1-\sin \delta}$

It is a quadratic algebraic constraint: the entries of $T$ take values on a coneyield surface, see Figure 4 . One can note that for metals, unlike granular materials, the yield surface is a topological cylinder (Han and Reddy, 1999). Here, the constitutive relations are homogeneous of degree zero in the elements of the strain rate tensor (Jackson, 1983) which reflects the very unintuitive behavior of granular materials.

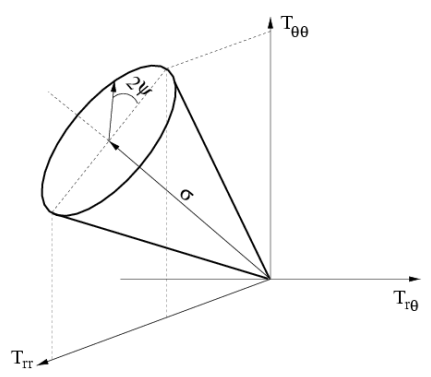

Fig. 4. Yield surface corresponding to (3.6). The variables $\sigma$ and $\psi$ are the Sokolovskii variables.

The resolution of the above system of equations presents in general formidable mathematical and numerical difficulties. In some special cases however, solutions can be constructed. In the early 1960 's, Jenike (1961), discovered similarity solutions for the steady state equations governing the flow of granular materials under gravity in a conical or wedge-shaped hopper, see Figure 5. In these solutions, particle paths are radial lines converging on the vertex of the hopper. For this reason, the solutions are referred to as radial solutions. The similarity is reflected in scalings of the stress and velocity with respect to radial distance $r$. Namely, those similarity solutions are such that generic stress and velocity entries $T_{i j}$ and $v_{i}$ satisfy 


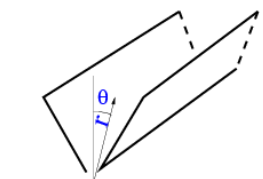

wedge-shaped hopper

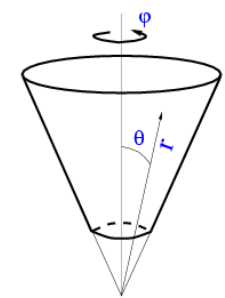

conical hopper
Fig. 5. Geometry and coordinate systems for the two dimensional, wedge-shaped hopper and the three dimensional conical hopper.

$T_{i j}(r, \theta, \phi)=r T_{i j}^{0}(\theta)$,

$v_{i}(r, \theta, \phi)=\frac{1}{r^{2}} v_{i}^{0}(\theta)$,

where $(r, \theta, \phi)$ refers to a spherical coordinate system with origin at the vertex of the hopper. The stress decreases along particle paths while the particles accelerate. Radial solutions form the basis for much of the work on the design of mass flow hoppers, in which the flow is thought to be approximately radial. The stability of the above solutions has been analyzed by Pitman (1988) and Gremaud et al., 2000. Their use has been generalized to axisymmetric domains with inserts (Gremaud et al., 2000). The generalization of Jenike's solutions to non axisymmetric pyramidal domains ${ }^{4}$ has proved surpringly challenging. This seemingly routine generalization has lead to extremely interesting results. For instance, breaking the axial symmetry implies secondary circulation. In short, Jenike's does not generalize to that case. Further, new resonance phenomena have been brought to the fore, see Gremaud et al. 2002.

Similarity solutions can only be considered as rough approximations in most

4 Those are domains invariant under transformations of the type $r \mapsto c r$ where the radial spherical coordinate. practical cases where no "invariance" in the radial direction exists for most containers. The full system of equations $(2.1,3.5,3.6)$, together with appropriate constitutive equations and boundary conditions has thus to be studied and solved. The system is differential algebraic in nature and unlike, say, problems in fluid dynamics, there is no obvious way to solve the algebraic constraint (3.6). Even though the topic of differential algebraic equations (DAE) has received a lot of attention in the ODE community (Brenan et al., 1996), most of the successful theoretical and numerical ideas developed there are just starting to be migrated to the more challenging cases of partial differentialalgebraic equations (PDAEs) (Martinson and Barton, 2000).

The following problem can be considered as an illustrative example of the difficulties attached to solving $(2.1,3.5$, 3.6) and similar problems ${ }^{5}$

$$
\begin{aligned}
\partial_{y} U+\partial_{x} V & =0, \\
U^{2}+V^{2} & =1 .
\end{aligned}
$$

Three main types of methods can be considered: (i) Parametrization of the manifold describing the constraint: the algebraic constraint is solved and its solution plugged into the PDE. (ii) Introduction of "stream functions": the PDE is "solved" and its solution plugged into the algebraic constraint. (iii) Use of existing DAE techniques such as constraint stabilization: both the algebraic and differential parts of the problem are kept.

Parametrization of the manifold corresponding to the yield surface is by

\footnotetext{
5 Without loss of generality, one can consider the case of vanishing right hand for both (2.1) and (3.7), through an adequate change of variables.
} 
far the most common approach and we focus on it in this exposition. Suitable local coordinates are introduced to solve the equations on that manifold (Potra and Rheinboldt, 1990). For (3.7, 3.8), one would set $U=\cos \psi$ and $V=\sin \psi$, for instance. In the case of the full problem $(2.1,3.5,3.5)$, similar parametrizations (Sokolovskii variables) can be found (Sokolovskii, 1965, Nedderman, 1992), see Figure 4. It should be noted however that such a reformulation destroys the conservation form of the equations, preventing any possibility of reliably analyzing or computing any nonsmooth solutions.

Another possibility consists in solving the algebraic equation, that is representing the yield surface as a graph rather than a parametric surface. The advantage is here that conservation form is preserved. In Gremaud and Matthews, 2000 and 2001, and Matthews, 2000, this approach was followed successfully in conjunction with the use of a discontinuous Galerkin method, see Cockburn and Shu, 1998, in the references therein. The application of this method to three dimensional hopper flows is illustrated in Figure 6.

For both approaches, parametric surface and graph, it may not be possible to use the same parametrization throughout the whole integration. For instance, solving for $V$ from (3.8) and then plugging into (3.7) reveals some general problems. First, one may get more than one solution. For the full problem, this corresponds to the existence of passive and active states (Nedderman, 1992). Second, as can be clearly from $(3.7,3.8)$, the speed of propagation can be unbounded.

Our calculations have brought to the fore significant difficulties attached to
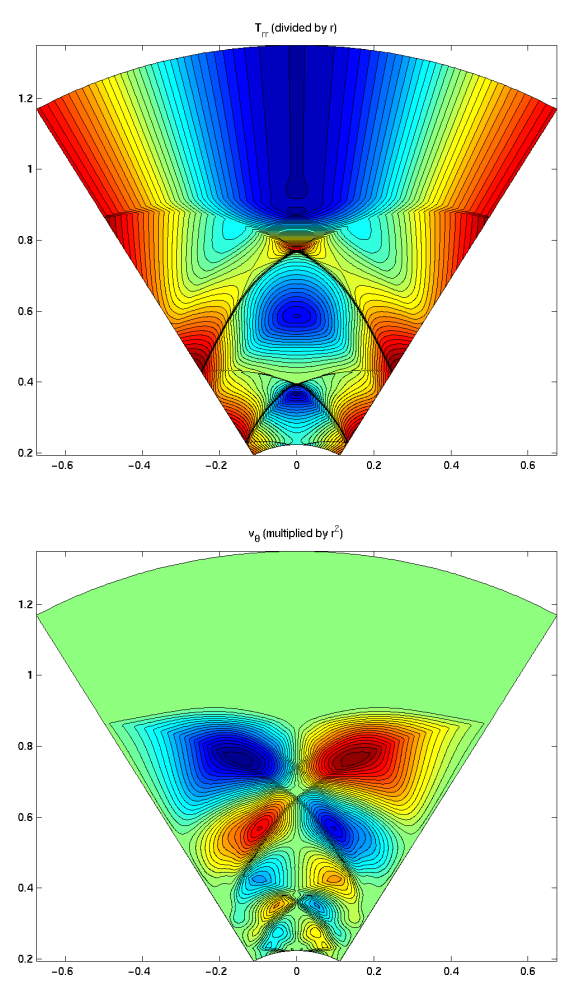

Fig. 6. An incompressible, perfectly plastic Mohr-Coulomb material is flowing under gravity in a conical hopper with a half-opening angle of 30 degrees. The wall of the hopper switches from rougher to smoother a third of the way down. Spherical coordinates are used. Top: $T_{r r}$, bottom: $v_{\theta}$.

the standard model $(2.1,3.5,3.6)$. For instance, the precise nature of the flow rule for a granular material has been the object of some controversy for a long time (Jackson, 1983). The issue is whether principal stresses and principal strain rates are aligned. Some models allow for some missalignment (de Josselin de Jong, 1971, 1977, Spencer, 1986, 1997) while some others do not (Savage and Sayed, 1979, Jackson, 1983, Jenike, 1961, 1987, Schaeffer, 1987, Nedderman, 1992. Although, there appears to be convincing experimental evidences of some missalignment taking place, see e.g. Drescher, 1976, as well as our results on Figure 7, whether 


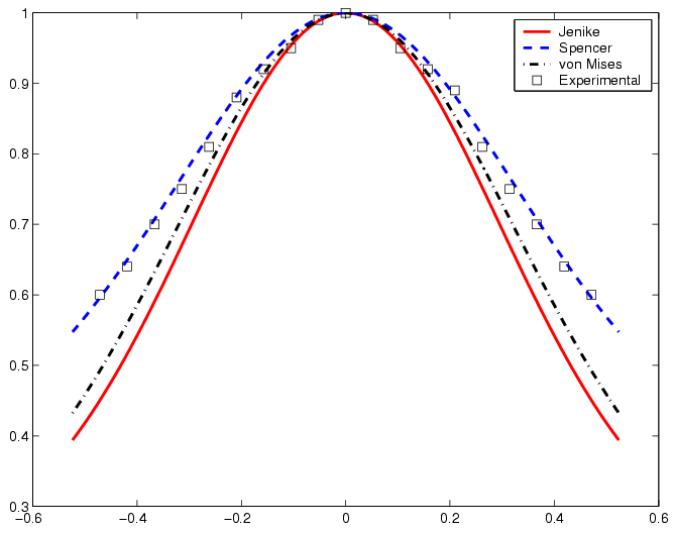

Fig. 7. Comparison of the prediction for the radial velocity profile (similarity solution) between the predictions obtained using a Tresca yield condition (curve labeled "Jenike", essentially (3.6)), a von Mises yield condition, Spencer's model (Spencer, 1986, 1997) and experimental values taken from Cleaver and Nedderman, 1992, Figure [8]. The experimental setup is kale seeds in an aluminum hopper (angles are in radians).

it is of the kind predicted by the above mentioned models is not clear at all. Further, those models typically require a very high degree of symmetry (at least axial symmetry); we are aware of no fully three-dimensional model allowing missalignment.

In some cases, calculations (Matthews, 2000, Gremaud and Matthews, 2001) have shown the standard model to lead to unphysical solutions. Depending on the boundary conditions, solutions may locally be in states that are not energetically acceptable (Harris, 1986). Careful analysis has shown those phenomena not to be numerical artifacts ${ }^{6}$. To circumvent those difficulties, we are in the process of studying the full plasticity problem $(2.1,3.5)$ with a von Mises condition instead of the more usual Tresca condition, see Nedderman, 1992, Gremaud et al., 2002. In many, but not all, cases of interest the corresponding system is elliptic (Schaeffer, 1987), simplifying greatly the handling of boundary conditions. However, the stress problem does not decouple and all variables have to be solved for at once.

\section{Acknowledgments}

This paper synthesizes previously unpublished results obtained in collaboration with Robbie Buckingham, Chris Kuster, Matt Matthews and David Schaeffer.

\section{References}

[1] Ahmed, S., Buckingham, R., Gremaud, P.A. , Hauck, C., Kuster, C., Prodanovic, M., Royal, T. and Silantyev, V., On the Shape of Sandpiles around Obstacles, Proceedings of the 8th Industrial Mathematics Modeling Workshop for Graduate Students at the Center for Re-

\footnotetext{
6 The system $(2.1,3.5,3.6)$ corresponds to two pairs of nonlinear hyperbolic conservation laws. We hypothesize that the above admissibility issue is related to the difficulty of imposing physically relevant boundary conditions to such a propagation problem. Elementary physical arguments show that in addition to the obvious conditions on the wall of the hopper, boundary conditions should be imposed both at the bottom and the top of the hopper, that is, part of the information propagates upwards and part of it downwards. Various combinations of conditions were tried by Matthews (2000); none of those combinations were found to be acceptable for a wide range of parameters.
} 
search in Scientific Computation, North Carolina State University, July 2002, in preparation.

[2] Bagnold, R.A., 1941. The physics of blown sand and desert dunes. Metheun.

[3] Behringer, R.P., 1993. The dynamics of flowing sand. Nonlinear Sci. Today, 3: $1-15$.

[4] Bouchaud, J.-P., Cates, M.E., Ravi Prakash, J. and Edwards, S.F., 1995. Hysteresis and metastability in a continuum sandpile model. Phys. Rev. Lett., 74: 1982-1985.

[5] Boutreux, T, Raphaël, E. and de Gennes, P.G., 1997. Propagation of a pressure step in a granular material: the role of wall friction. Phys. Rev. E, 55: 5759-5773.

[6] Brenan, K.E., Campbell, S.L. and Petzold, L.R., 1996. Numerical Solution of Initial-Value Problems in Differential-Algebraic Equations, SIAM.

[7] Cleaver, J.A.S. and Nedderman, R.M., 1993. Measurement of velocity profiles in conical hoppers, Chem. Eng. Sc., 48: 3703-3712.

[8] Cockburn, B. and Shu, C.W., 1998. The Runge-Kutta discontinuous Galerkin method for conservation laws V: multidimensional systems, J. Comput. Phys., 141: 199224.

[9] Coulomb, C.A., 1776. Essai sur une application des règles de maximis et minimis à quelques problèmes de statique relatifs à l'architecture. Mémoires de Mathématique de l'Académie des Sciences, Paris, 7: 343-382.

[10] Crandall, M.G. and Lions, P.L., 1983. Viscosity solutions of Hamilton-Jacobi equations. Trans. Amer. Math. Soc., 277: 1-42.

[11] Crandall, M.G. and Lions, P.L., 1984. Two approximations of solu- tions of Hamilton-Jacobi equations. Math. Comp., 43: 1-19.

[12] Crandall, M.G., Evans, L.C. and Lions, P.L., 1984. Some properties of viscosity solutions of HamiltonJacobi equations. Trans. Amer. Math. Soc., 282: 487-502.

[13] Dijkstra, E.W., 1959. A note on two problems in connection with graphs. Nümerische Mathematik, 1 : 269-271.

[14] de Josselin de Jong, G., 1971. The double-sliding, free rotating model for granular assemblies. Géotechnique, 21: 155-163.

[15] de Josselin de Jong, G., 1977. Mathematical elaboration of the double-sliding, free-rotating model. Archs. Mech., 29: 561-591.

[16] Drescher, A., 1976. An experimental investigation of flow rules for granular materials using optically sensitive glass particles. Géotechnique, 26: 591-601.

[17] Feldman, M., 1999. Growth of a sandpile around an obstacle, in Monge Ampère Equation: Applications to Geometry and Optimization, L.A. Caffarelli, M. Milman Eds., Contemporary Mathematics, \# 226: 55-78.

[18] Grasselli, Y. and Herrmann, H.J., 1999. Shapes of heaps and in silos. Eur. Phys. J.B, 10: 673-679.

[19] Grasselli, Y. and Herrmann, H.J., 2001. Crater formation on a three dimensional granular heap. Granular Matter, 3: 201-204.

[20] Gremaud, P.A. and Matthews, J.V., 2000. Simulation of gravity flow of granular materials in silos, in Discontinuous Galerkin Methods, Theory, Computation and Applications, B. Cockburn, G.E. Karniadakis, C.-W. Shu Eds. Lectures Notes in Computational Science and Engineering \#11: 125-134. 
[21] Gremaud, P.A. and Matthews, J.V., 2001. On the computation of hopper flows:I, Stress determination for Coulomb materials. J. Comput. Phys., 166: 63-83.

[22] Gremaud, P.A., Matthews, J.V. and Shearer, M., 2000. Similarity solutions for granular materials in hoppers, in: Nonlinear PDE's, dynamics, and continuum physics, J. Bona, K. Saxton and R. Saxton Eds. Contemporary Mathematics, AMS, \#255: 79-95.

[23] Gremaud, P.A., Matthews, J.V. and Schaeffer, D.G., 2002. Secondary circulation in granular flow through nonaxisymmetric hoppers. Center for Research in Scientific Computation, NCSU, technical report CRSC-TR02-29, submitted to SIAM J. Appl. Math.

[24] Gremaud, P.A., Schaeffer, D.G. and Shearer, M., 2000. Numerical Determination of Flow Corrective Inserts for Granular Materials in Conical Hoppers. Int. J. Nonlinear Mech., 35: 869-882.

[25] Han, W. and Reddy, B.D., 1999. Plasticity; Mathematical Theory and Numerical Analysis. Springer.

[26] Harris, D., 1986. On the energy dissipation and velocity discontinuities in granular materials and solution of a boundary value problem in geophysics. Acta Mechanica, 64: 63-76.

[27] Heyman, J., 1972. Coulomb's memoir on statics. Cambridge University Press.

[28] Iserles, A. and Strang, G., 1983. The optimal accuracy of difference schemes. Trans. AMS, 277: 779-803.

[29] Jackson, R., 1983. Some mathematical and physical aspects of continuum models for the motion of granular materials, in: Theory of Dispersed Multiphase Flow, R.E. Meyer Ed. Academic Press,
291-337.

[30] Jenike, A.W., 1961. Gravity flow of bluk solids. Bulletin No. 108, Utah Eng. Expt. Station, University of Utah, Salt Lake City.

[31] Jenike, A.W., 1987. A theory of flow of particulate solids in converging and diverging channels based on a conical yield function. Powder Tech., 50: 229-236.

[32] Jiang, G.S. and Peng, D., 2000. Weigthed ENO schemes for Hamilton-Jacobi equations. SIAM J. Sci. Comput., 21: 2126-2143.

[33] Kapoor, S. , Maheshwari, S.N. and Mitchell, J.S.B., 1997. An efficient algorithm for euclidean shortest paths among polygonal obstacles in the plane. Discrete Comput. Geom., 18: 377-383.

[34] Martinson, W.S. and Barton, P.I., 2000. A Differentiation Index for Partial Differential-Algebraic Equations. SIAM J. Sci. Comput., 21; 2295-2315.

[35] Matthews, J.V., 2000. An Analytical and Numerical Study of Granular Flows in Hoppers. Ph.D. Thesis, North Carolina State University, Department of Mathematics.

[36] Nedderman, R.M., 1992. Static and kinematic of granular materials. Cambridge University Press.

[37] Osher, S. and Shu, C.W., 1991. High order essentially nonoscillatory schemes for Hamilton-Jacobi equations. SIAM J. Numer. Anal., 28: 907-922.

[38] Pitman, E.B., 1988. The stability of granular flow in converging hoppers. SIAM J. Appl. Math., 48: 1033-1052.

[39] Potra, F.A. and Rheinboldt, W.C., 1990. Differential-geometric techniques for solving differential algebraic equations, in Real-Time Integration of Mechanical System Sim- 
ulation, E.J. Haug and R.C. Deyo, eds, Springer.

[40] Qian, J. and Symes, W.W., 2002. An adaptive finite difference method for traveltimes and amplitudes. Geophysics, 67: 167-176.

[41] Rouy, E. and Tourin, A., 1992. A viscosity solutions approach to shape-from-shading. SIAM J. Numer. Anal., 29: 867-884.

[42] Savage, S.B. and Sayed, M., 1979. Gravity flow of cohesionless granular materials in wedge-shaped hoppers, in Mechanics Applied to the Transport of Bulk Materials, S.C Cowin ed., American Society of Mechanical Engineers, 1-24.

[43] Schaeffer, D.G., 1987. Instability in the evolution equations describing incompressible granular flow. J. Diff. Eq., 66: 19-50.

[44] Sethian, J.A., 1999. Fast marching methods. SIAM Review, 41: 199235.

[45] Sethian, J.A. and Popovici, A.M., 1999. 3-D traveltime computation using the fast marching method. Geophysics, 64: 516-523.

[46] Sokolovskii, V.V., 1965. Statics of granular media. Pergamon Press.

[47] Spencer, A.J.M., 1986. Instability of steady shear flow of granular materials. Acta Mechanica, 64: 77-87.

[48] Spencer, A.J.M., 1997. Remarks on coaxiality in fully developed gravity flows of dry granular materials, in: IUTAM Symposium on Mechanics of Granular and Porous Materials, N.A. Fleck and A.C.F. Cocks Eds,Kluwer, 227-238.

[49] Tsai, Y.H.R, Cheng,L.T., Osher, S. and Zhao, H.K., 2002. Fast sweeping algorithms for a class of HamiltonJacobi equations. preprint, http://www.math.uci.edu/ zhao.

[50] Tsitsiklis, J., 1995. Efficient algo- rithms for globally optimal trajectories. IEEE Transactions on Automatic Control, 40: 1528-1538.

[51] van Trier, J. and Symes, W.W., 1991. Upwind finite difference of traveltimes. Geophysics, 56: 812821.

[52] Zhao, H.K., 2002. Fast sweeping method for Eikonal equations I: Distance function. preprint, http://www.math.uci.edu/ zhao. 